\title{
The Application of Special Hermite Finite Elements Coupled with Collocation to the 3D Poisson Equation
}

\author{
Lidiya Gileva, ${ }^{1,2, a)}$ Evgenya Karepova, ${ }^{1,2, b)}$ and Vladimir Shaidurov ${ }^{1,2, c)}$ \\ ${ }^{1)}$ Institute of Computational Modelling of SB RAS, 660036, Akademgorodok, Krasnoyarsk, Russia. \\ ${ }^{2)}$ Siberian Federal University, 79 Svobodny pr., 660041 Krasnoyarsk, Russia. \\ a)Electronic mail: gileva@icm.krasn.ru

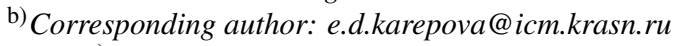 \\ ${ }^{c)}$ Electronic mail: shaidurov04@mail.ru
}

\begin{abstract}
A new $C^{0}$ Hermite element of degree 3 on a cuboid is proposed for the three-dimensional Poisson equation. A feature of the element is that it allows one to apply the collocation technique. As a result, the dimension of the system of equations is reduced without deterioration of the convergence order. Numerical results confirm high efficiency of the proposed method.
\end{abstract}

\section{INTRODUCTION}

$C^{0}$ Hermite finite elements can be applied when solving second order partial differential equations with a sufficiently smooth solution. Contrary to fourth order equations, in this case a weak solution is not required to have continuous first order derivatives.

The construction of $C^{0}$ Hermite elements is easier in comparison with $C^{1}$ ones. Examples of the most popular Hermite finite elements can be found in [1]. The Bogner-Fox-Schmit rectangle [2] seems to be the simplest version of $C^{1}$ finite element in 2D. In [3, 4] 2D and 3D $C^{1}$ finite elements of the Bogner-Fox-Schmit type are proposed and studied. The advantage of rectangular elements in comparison with simplicial ones is that their basis functions are tensor products of one-dimensional polynomials. So, the simplicial $C^{1}$ finite elements are of still more complicated structure and often are difficult to implement even in 2D (see, for example, the Argyris triangle [1,5]). In 3D, the development of simplicial finite elements presents severe difficulties [6]. In [7] some $C^{1}$ finite elements of high degree on tetrahedron are constructed and their approximation properties are studied. In [8] composite $C^{1}$ triangular finite elements (macroelements) are discussed and a composite $C^{1}$ tetrahedral element is proposed.

In comparison with Lagrange finite elements, Hermite finite elements of the same degree (and, hence, providing the same convergence order) result in a system of linear algebraic equations of considerably smaller dimension [9]. In [10, 11] we propose and study a two-dimensional rectangular Hermite element, which permits the collocation technique for some equations. Requiring the residual to vanish at the nodes, we can express one degree of freedom in terms of other ones from the original equation and eliminate the related equations from the discrete system. This enables one to reduce still further the dimension of the system of equations without reducing the order of convergence.

In the paper, we generalize this approach to the three-dimensional Poisson equation. We propose a $C^{0}$ Hermite element of degree 3 on a cuboid, whose degrees of freedom involve the values of a function and its second-order derivatives with respect to each variable at the vertices of the cuboid. To construct the reduced system, we eliminate the unknowns being the values of the second order derivative with respect to one variable. In addition, we perform some elementary manipulations to bring the matrix of the reduced system to symmetric form. We also propose an auxiliary Hermite element of lower degree with the following property: the matrix of the system of the finite element method for the auxiliary element coincides with the symmetrized matrix of the reduced system. This proves that the matrix of the reduced system is nonsingular and simplifies the calculation of its entries.

Numerical experiments demonstrate that the elimination of a part of unknowns and, as a result, reducing the dimension of the system of equations by a factor of $1 / 4$ retain the order of convergence of an approximate solution.

\section{NOTATIONS}

Let $\Omega=\left(0, H_{1}\right) \times\left(0, H_{2}\right) \times\left(0, H_{3}\right) \subset R^{3}$ be a cuboid with the boundary $\Gamma$. To construct a uniform partition $\mathscr{T}_{h}$, we subdivide $\bar{\Omega}$ into $N_{1} \times N_{2} \times N_{3}$ closed cuboids by the planes

$$
x=i h_{1}, \quad y=j h_{2}, \quad z=k h_{3}, \quad i=0, \ldots, N_{1}, \quad j=0, \ldots, N_{2}, \quad k=0, \ldots, N_{3}
$$


where $h_{s}=H_{s} / N_{s}, s=1,2,3$. Denote $h=\max \left\{h_{1}, h_{2}, h_{3}\right\}$.

For functions $u, v$ defined and measurable on $\Omega$, introduce the inner product and the induced norm:

$$
(u, v)=\int_{\Omega} u v d \Omega, \quad|u|_{0, \Omega}=(u, u)^{1 / 2} .
$$

Denote by $L_{2}(\Omega)$ the space of all functions $u$ defined and measurable on $\Omega$ with the finite norm $|u|_{0, \Omega}$. Introduce also the semi-norm

$$
|u|_{s, \Omega}=\left(\sum_{i+j+k=s}\left|\frac{\partial^{s} u}{\partial x^{i} \partial y^{j} \partial z^{k}}\right|_{0, \Omega}^{2}\right)^{1 / 2} .
$$

Denote by $H^{s}(\Omega)$ the space of all functions $u$ defined and measurable on $\Omega$ together with their partial derivatives up to order $s$ with the finite norm [12]

$$
\|u\|_{s, \Omega}=\left(|u|_{0, \Omega}^{2}+|u|_{1, \Omega}^{2}+\ldots+|u|_{s, \Omega}^{2}\right)^{1 / 2} .
$$

Denote by $P_{k}$ the space of all polynomials in three variables up to degree $s$ :

$$
\sum_{0 \leq i+j+k \leq s} a_{i, j, k} x^{i} y^{j} z^{k}
$$

Define a "reference" finite element as a triple $(\hat{e}, \hat{P}, \hat{\Sigma})$ where $\hat{e}$ is a "reference" cell, $\hat{P}$ is a space of polynomials defined on $\hat{e}$, and $\hat{\Sigma}$ is a set of functionals called degrees of freedom (DoF).

\section{A THREE-DIMENSIONAL CUBIC HERMITE ELEMENT}

In $[10,11]$ we present a two-dimensional cubic Hermite element with second-order derivatives in the set of DoF. Now we propose its three-dimensional version.

The "reference" element is defined as follows:

$$
\begin{aligned}
\hat{e}= & {[0,1]^{3}, } \\
\hat{P}= & \operatorname{span}\left\{1, \hat{x}, \hat{y}, \hat{z}, \hat{x}^{2}, \hat{y}^{2}, \hat{z}^{2}, \hat{x} \hat{y}, \hat{y} \hat{z}, \hat{x} \hat{z}, \hat{x}^{3}, \hat{y}^{3}, \hat{z}^{3}, \hat{x}^{2} \hat{y}, \hat{x} \hat{y}^{2}, \hat{y}^{2} \hat{z}, \hat{y} \hat{z}^{2}, \hat{x}^{2} \hat{z}, \hat{x} \hat{z}^{2}, \hat{x} \hat{y} \hat{z}, \hat{x}^{3} \hat{y}, \hat{x}^{3} \hat{z}, \hat{x}^{2} \hat{y} \hat{z}, \hat{x} \hat{y}^{3}, \hat{y}^{3} \hat{z},\right. \\
& \left.\hat{x} \hat{y}^{2} \hat{z}, \hat{x} \hat{z}^{3}, \hat{y} \hat{z}^{3}, \hat{x} \hat{y} \hat{z}^{2}, \hat{x} \hat{y} \hat{z} \hat{z}, \hat{x} \hat{y}^{3} \hat{z}, \hat{x} \hat{y} \hat{z}^{3}\right\}, \\
\hat{\Sigma}= & \left\{\hat{\psi}_{i}^{0}(p)=p\left(\hat{a}_{i}\right), \hat{\psi}_{i}^{1}(p)=p_{x x}\left(\hat{a}_{i}\right), \hat{\psi}_{i}^{2}(p)=p_{y y}\left(\hat{a}_{i}\right), \hat{\psi}_{i}^{3}(p)=p_{z z}\left(\hat{a}_{i}\right), i=1, \ldots, 8, \forall p \in \hat{P}\right\} .
\end{aligned}
$$

where $\hat{a}_{i}, i=1, \ldots, 8$ are the nodes of the element which coincide with the vertices of $\hat{e}$ and are numbered as shown in Fig. 1. Observe that $\hat{P} \supset P_{3}, \operatorname{dim} \hat{P}=\operatorname{card} \hat{\Sigma}=32$.

It is easy to verify that the Lagrange basis of element (1) is of the form

$$
\begin{array}{llll}
\hat{\varphi}_{1}^{0}:=(1-\hat{x})(1-\hat{y})(1-\hat{z}) ; & \hat{\varphi}_{3}^{0}:=\hat{x} \hat{y}(1-\hat{z}) ; & \hat{\varphi}_{5}^{0}:=(1-\hat{x})(1-\hat{y}) \hat{z} ; & \hat{\varphi}_{7}^{0}:=\hat{x} \hat{y} \hat{z} ; \\
\hat{\varphi}_{1}^{1}:=\lambda(\hat{x})(1-\hat{y})(1-\hat{z}) ; & \hat{\varphi}_{3}^{1}:=\mu(\hat{x}) \hat{y}(1-\hat{z}) ; & \hat{\varphi}_{5}^{1}:=\lambda(\hat{x})(1-\hat{y}) \hat{z} ; & \hat{\varphi}_{7}^{1}:=\mu(\hat{x}) \hat{y} \hat{z} ; \\
\hat{\varphi}_{1}^{2}:=(1-\hat{x}) \lambda(\hat{y})(1-\hat{z}) ; & \hat{\varphi}_{3}^{2}:=\hat{x} \mu(\hat{y})(1-\hat{z}) ; & \hat{\varphi}_{5}^{2}:=(1-\hat{x}) \lambda(\hat{y}) \hat{z} ; & \hat{\varphi}_{7}^{2}:=\hat{x} \mu(\hat{y}) \hat{z} ; \\
\hat{\varphi}_{1}^{3}:=(1-\hat{x})(1-\hat{y}) \lambda(\hat{z}) ; & \hat{\varphi}_{3}^{3}:=\hat{x} \hat{y} \lambda(\hat{z}) ; & \hat{\varphi}_{5}^{3}:=(1-\hat{x})(1-\hat{y}) \mu(\hat{z}) ; \hat{\varphi}_{7}^{3}:=\hat{x} \hat{y} \mu(\hat{z}) ; \\
\hat{\varphi}_{2}^{0}:=\hat{x}(1-\hat{y})(1-\hat{z}) ; & \hat{\varphi}_{4}^{0}:=(1-\hat{x}) \hat{y}(1-\hat{z}) ; & \hat{\varphi}_{6}^{0}:=\hat{x}(1-\hat{y}) \hat{z} ; & \hat{\varphi}_{8}^{0}:=(1-\hat{x}) \hat{y} z ; \\
\hat{\varphi}_{2}^{1}:=\mu(\hat{x})(1-\hat{y})(1-\hat{z}) ; & \hat{\varphi}_{4}^{1}:=\lambda(\hat{x}) \hat{y}(1-\hat{z}) ; & \hat{\varphi}_{6}^{1}:=\mu(\hat{x})(1-\hat{y}) \hat{z} ; & \hat{\varphi}_{8}^{1}:=\lambda(\hat{x}) \hat{y} \hat{z} ; \\
\hat{\varphi}_{2}^{2}:=\hat{x} \lambda(\hat{y})(1-\hat{z}) ; & \hat{\varphi}_{4}^{2}:=(1-\hat{x}) \mu(\hat{y})(1-\hat{z}) ; \hat{\varphi}_{6}^{2}:=\hat{x} \lambda(\hat{y}) \hat{z} ; & \hat{\varphi}_{8}^{2}:=(1-\hat{x}) \mu(\hat{y}) \hat{z} ; \\
\hat{\varphi}_{2}^{3}:=\hat{x}(1-\hat{y}) \lambda(\hat{z}) ; & \hat{\varphi}_{4}^{3}:=(1-\hat{x}) \hat{y} \lambda(\hat{z}) ; & \hat{\varphi}_{6}^{3}:=\hat{x}(1-\hat{y}) \mu(\hat{z}) ; & \hat{\varphi}_{8}^{3}:=(1-\hat{x}) \hat{y} \mu(\hat{z})
\end{array}
$$

where $\lambda(t):=t(1-t)(t-2) / 6, \mu(t):=t\left(t^{2}-1\right) / 6$.

For the partition $\mathscr{T}_{h}$, the usual affine mapping of the "reference" element onto an elementary cell $\left[x_{i}, x_{i}+h_{1}\right] \times$ $\left[y_{j}, y_{j}+h_{2}\right] \times\left[z_{k}, z_{k}+h_{3}\right]$ is of the form

$$
x=x_{i}+h_{1} \hat{x}, \quad y=y_{j}+h_{2} \hat{y}, \quad z=z_{k}+h_{3} \hat{z} .
$$




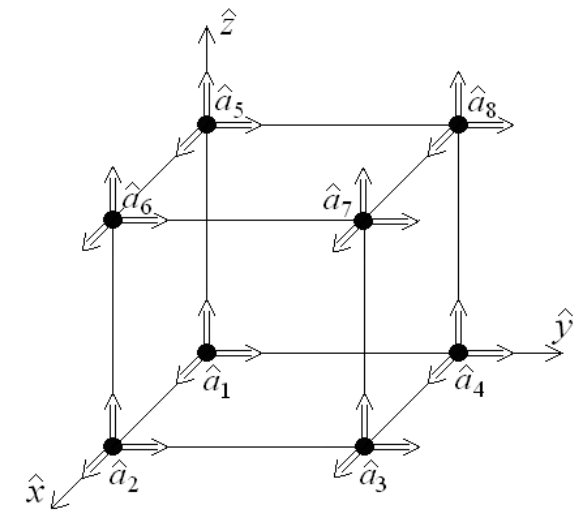

FIGURE 1. The "reference" element. A double arrow shows the DoF being the value of the second-order derivative in the corresponding direction.

Since $\hat{P} \supset P_{3}$, for the interpolant $\tilde{u}_{I}$ of a function $u \in H^{4}(\Omega)$ on the partition $\mathscr{T}_{h}$, we have the usual accuracy estimates $[1,13]$

$$
\begin{array}{r}
\left|u-\tilde{u}_{I}\right|_{0, \Omega} \leq c h^{4}\|u\|_{4, \Omega}, \\
\left\|u-\tilde{u}_{I}\right\|_{s, \Omega} \leq c h^{4-s}\|u\|_{4, \Omega}, \quad s=1,2
\end{array}
$$

which define the convergence order of an approximate solution. From here on, $c$ denotes a positive constant independent of $u$ and $h$.

As in the case of the two-dimensional version, finite element (1) enables one to apply the collocation technique for some partial differential equations, in particular, for the Poisson equation. This results in reducing the dimension of the discrete problem.

\section{A DISCRETE PROBLEM}

Without loss of generality, for simplicity sake we assume that $\Omega=(0,1)^{3}$. Consider the problem

$$
\begin{array}{r}
-\Delta u=f \quad \text { in } \Omega, \\
u=0 \quad \text { on } \Gamma
\end{array}
$$

where $\Delta=\partial^{2} / \partial x^{2}+\partial^{2} / \partial y^{2}+\partial^{2} / \partial z^{2}$ is the Laplacian, $f \in H^{2}(\Omega)$.

For (4)-(5) we construct the standard scheme of the finite element method using Hermite element (1). Notice that we have $N_{1}=N_{2}=N_{3}=N, h_{1}=h_{2}=h_{3}=h$. An approximate solution of (4)-(5) has the form

$$
\begin{aligned}
\tilde{u}^{h} & =\sum_{i, j, k=1}^{N-1} \tilde{u}^{h}\left(\omega_{i, j, k}\right) \varphi_{i, j, k}^{0}(x, y, z) \\
& +h^{2} \sum_{i, j, k=0}^{N}\left(\tilde{u}_{x x}^{h}\left(\omega_{i, j, k}\right) \varphi_{i, j, k}^{1}(x, y, z)+\tilde{u}_{y y}^{h}\left(\omega_{i, j, k}\right) \varphi_{i, j, k}^{2}(x, y, z)+\tilde{u}_{z z}^{h}\left(\omega_{i, j, k}\right) \varphi_{i, j, k}^{3}(x, y, z)\right)
\end{aligned}
$$

where $\omega_{i, j, k}=\left(x_{i}, y_{j}, z_{k}\right), x_{i}=i h, y_{j}=j h, z_{k}=k h$; the unknown coefficients $\tilde{u}^{h}\left(\omega_{i, j, k}\right), \tilde{u}_{x x}^{h}\left(\omega_{i, j, k}\right), \tilde{u}_{y y}^{h}\left(\omega_{i, j, k}\right)$, and $\tilde{u}_{z z}^{h}\left(\omega_{i, j, k}\right)$ are the values of the approximate solution and the approximate values of the second-order derivatives of the solution of (4)-(5) at the grid nodes, respectively. The basis functions $\varphi_{i, j, k}^{\alpha}(x, y, z), \alpha=0, \ldots, 3$ of the finite element space are obtained from basis functions (2) of the "reference" element with the help of the affine mapping of the form (3).

For the unknown coefficients of (6) we have a system of linear algebraic equations. The number of equations as well as the number of unknowns equals to $4 N_{\text {int }}$ where $N_{\text {int }}=(N-1)^{3}$ is the number of interior nodes. The values of $\tilde{u}^{h}, \tilde{u}_{x x}^{h}, \tilde{u}_{y y}^{h}$, and $\tilde{u}_{z z}^{h}$, at the boundary nodes coincide with those of the exact solution and are determined from (4)-(5). 
The matrix of the system can be written in a block form

$$
A=\left(\begin{array}{llll}
A_{00} & A_{01} & A_{02} & A_{03} \\
A_{10} & A_{11} & A_{12} & A_{13} \\
A_{20} & A_{21} & A_{22} & A_{23} \\
A_{30} & A_{31} & A_{32} & A_{33}
\end{array}\right)
$$

where $A_{\alpha, \beta}, \alpha, \beta=0, \ldots, 3$ are $N_{\text {int }} \times N_{\text {int }}$ blocks with the entries

$$
\left(A_{\alpha, \beta}\right)_{i, j, k, p, q, s}=a\left(\varphi_{p, q, s}^{\beta}, \varphi_{i, j, k}^{\alpha}\right) \quad i, j, k, p, q, s=1, \ldots, N-1
$$

and the bilinear form in the right-hand side of (8) is as follows:

$$
a(v, w)=\int_{\Omega}\left(v_{x} w_{x}+v_{y} w_{y}+v_{z} w_{z}\right) d \Omega
$$

Observe that $A_{\alpha, \beta}=A_{\beta, \alpha}^{T}$, i.e., matrix (7) is symmetric.

Thus, the system of the finite element method consists of four groups of equations

$$
\begin{aligned}
& A_{00} U_{0}^{h}+A_{01} U_{1}^{h}+A_{02} U_{2}^{h}+A_{03} U_{3}^{h}=F_{0}^{h}, \\
& A_{10} U_{0}^{h}+A_{11} U_{1}^{h}+A_{12} U_{2}^{h}+A_{13} U_{3}^{h}=F_{1}^{h}, \\
& A_{20} U_{0}^{h}+A_{21} U_{1}^{h}+A_{22} U_{2}^{h}+A_{23} U_{3}^{h}=F_{2}^{h}, \\
& A_{30} U_{0}^{h}+A_{31} U_{1}^{h}+A_{32} U_{2}^{h}+A_{33} U_{3}^{h}=F_{3}^{h}
\end{aligned}
$$

where $U_{0}^{h}=\left\{\tilde{u}^{h}\left(\omega_{p, q, s}\right)\right\}_{p, q, s=1}^{N-1}, U_{1}^{h}=\left\{h^{2} \tilde{u}_{x x}^{h}\left(\omega_{p, q, s}\right)\right\}_{p, q, s=1}^{N-1}, U_{2}^{h}=\left\{h^{2} \tilde{u}_{y y}^{h}\left(\omega_{p, q, s}\right)\right\}_{p, q, s=1}^{N-1}, U_{3}^{h}=\left\{h^{2} \tilde{u}_{z z}^{h}\left(\omega_{p, q, s}\right)\right\}_{p, q, s=1}^{N-1}$, $F_{\alpha}^{h}=\left\{\int_{\Omega} f \varphi_{i, j, k}^{\alpha} d \Omega\right\}_{i, j, k=1}^{N-1}, \alpha=0, \ldots, 3$. The entries of the matrices $A_{\alpha, \beta}, \alpha, \beta=0, \ldots, 3$ are given by (8)-(9). Notice that the equations corresponding to $i=1$ or $N-1, j=1$ or $N-1$, and $k=1$ or $N-1$ are modified in a usual way taking into account the boundary conditions.

Notice that in system (10)-(13) we use the $\operatorname{DoF} \psi_{i, j, k}^{0}\left(\tilde{u}^{h}\right)=\tilde{u}^{h}\left(\omega_{i, j, k}\right), \psi_{i, j, k}^{1}\left(\tilde{u}^{h}\right)=h^{2} \tilde{u}_{x x}^{h}\left(\omega_{i, j, k}\right), \psi_{i, j, k}^{2}\left(\tilde{u}^{h}\right)=$ $h^{2} \tilde{u}_{y y}^{h}\left(\omega_{i, j, k}\right)$, and $\psi_{i, j, k}^{3}\left(\tilde{u}^{h}\right)=h^{2} \tilde{u}_{z z}^{h}\left(\omega_{i, j, k}\right)$ of an approximate solution on an actual element $e \in \mathscr{T}_{h}$ as the unknowns, i.e., the approximate values of second-order derivatives are equipped with the factor $h^{2}$. This is convenient for several reasons, in particular, this improves properties of the stiffness matrix.

\section{THE ELIMINATION OF UNKNOWNS BY COLLOCATION}

Introduce the grid functions $u^{h}\left(\omega_{i, j, k}\right)=\tilde{u}^{h}\left(\omega_{i, j, k}\right), u_{x x}^{h}\left(\omega_{i, j, k}\right)=\tilde{u}_{x x}^{h}\left(\omega_{i, j, k}\right), u_{y y}^{h}\left(\omega_{i, j, k}\right)=\tilde{u}_{y y}^{h}\left(\omega_{i, j, k}\right)$, and $u_{z z}^{h}\left(\omega_{i, j, k}\right)=$ $\tilde{u}_{z z}^{h}\left(\omega_{i, j, k}\right), i, j, k=0, \ldots, N$. They satisfy the algebraic identity

$$
-u_{x x}^{h}\left(\omega_{i, j, k}\right)-u_{y y}^{h}\left(\omega_{i, j, k}\right)-u_{z z}^{h}\left(\omega_{i, j, k}\right)=f^{h}\left(\omega_{i, j, k}\right)-r^{h}\left(\omega_{i, j, k}\right)
$$

where $f^{h}$ is a grid function defined by $f^{h}\left(\omega_{i, j, k}\right)=f\left(\omega_{i, j, k}\right)$ and $r^{h}$ is the residual.

Assuming the residual is known, express $u_{z z}^{h}\left(\omega_{i, j, k}\right)$ from (14) and substitute it into (10)-(13). We obtain the system

$$
\begin{aligned}
& A_{00} U_{0}^{h}+\left(A_{01}-A_{03}\right) U_{1}^{h}+\left(A_{02}-A_{03}\right) U_{2}^{h}=F_{0}^{h}+h^{2} A_{03}\left(F^{h}-R^{h}\right), \\
& A_{10} U_{0}^{h}+\left(A_{11}-A_{13}\right) U_{1}^{h}+\left(A_{12}-A_{13}\right) U_{2}^{h}=F_{1}^{h}+h^{2} A_{13}\left(F^{h}-R^{h}\right), \\
& A_{20} U_{0}^{h}+\left(A_{21}-A_{23}\right) U_{1}^{h}+\left(A_{22}-A_{23}\right) U_{2}^{h}=F_{2}^{h}+h^{2} A_{23}\left(F^{h}-R^{h}\right), \\
& A_{30} U_{0}^{h}+\left(A_{31}-A_{32}\right) U_{1}^{h}+\left(A_{32}-A_{33}\right) U_{2}^{h}=F_{3}^{h}+h^{2} A_{33}\left(F^{h}-R^{h}\right)
\end{aligned}
$$

where $F^{h}=\left\{f^{h}\left(\omega_{i, j, k}\right)\right\}_{i, j, k=1}^{N-1}, R^{h}=\left\{r^{h}\left(\omega_{i, j, k}\right)\right\}_{i, j, k=1}^{N-1}$. As a result, the unknowns being the components of the vector $U_{3}^{h}$ are eliminated, but system (15)-(18) is overdetermined. In principle, we can neglect, for example, equations (18), but in this case we get system (15)-(17) with a non-symmetric matrix. 
The next step is to make the matrix of (15)-(17) symmetric with the help of (18). To this end, we subtract (18) from (16) and (17). This results in the equations

$$
\begin{aligned}
& \left(A_{10}-A_{30}\right) U_{0}^{h}+\left(A_{11}-A_{13}-A_{31}+A_{33}\right) U_{1}^{h}+\left(A_{12}-A_{13}-A_{32}+A_{33}\right) U_{2}^{h}=F_{1}^{h}-F_{3}^{h}+h^{2}\left(A_{13}-A_{33}\right)\left(F^{h}-R^{h}\right), \\
& \left(A_{20}-A_{30}\right) U_{0}^{h}+\left(A_{21}-A_{23}-A_{31}+A_{33}\right) U_{1}^{h}+\left(A_{22}-A_{23}-A_{32}+A_{33}\right) U_{2}^{h}=F_{2}^{h}-F_{3}^{h}+h^{2}\left(A_{23}-A_{33}\right)\left(F^{h}-R^{h}\right),
\end{aligned}
$$

respectively. Combining (15) and (19)-(20), we arrive at the reduced system with the symmetric matrix

$$
B=\left(\begin{array}{lll}
B_{00} & B_{01} & B_{02} \\
B_{10} & B_{11} & B_{12} \\
B_{20} & B_{21} & B_{22}
\end{array}\right)=\left(\begin{array}{lll}
A_{00} & A_{01}-A_{03} & A_{02}-A_{03} \\
A_{10}-A_{30} & A_{11}-A_{13}-A_{31}+A_{33} & A_{12}-A_{13}-A_{32}+A_{33} \\
A_{20}-A_{30} & A_{21}-A_{23}-A_{31}+A_{33} & A_{22}-A_{23}-A_{32}+A_{33}
\end{array}\right) .
$$

Now require that the residual vanish at the nodes and introduce grid functions $v^{h}, v_{x x}^{h}, v_{y y}^{h}$, and $v_{z z}^{h}$, being the values of a new approximate solution and new approximate values of the second-order derivatives of the exact solution of (4)-(5) at the nodes, respectively, which satisfy the equation

$$
-v_{x x}^{h}\left(\omega_{i, j, k}\right)-v_{y y}^{h}\left(\omega_{i, j, k}\right)-v_{z z}^{h}\left(\omega_{i, j, k}\right)=f^{h}\left(\omega_{i, j, k}\right), \quad i, j, k=0, \ldots, N .
$$

In addition, introduce the vectors $V_{0}^{h}=\left\{v^{h}\left(\omega_{i, j, k}\right)\right\}_{i, j, k=1}^{N-1}, V_{1}^{h}=\left\{v_{x x}^{h}\left(\omega_{i, j, k}\right)\right\}_{i, j, k=1}^{N-1}, V_{2}^{h}=\left\{v_{y y}^{h}\left(\omega_{i, j, k}\right)\right\}_{i, j, k=1}^{N-1}$ and $V_{3}^{h}=\left\{v_{z z}^{h}\left(\omega_{i, j, k}\right)\right\}_{i, j, k=1}^{N-1}$. In (15) and (19)-(20) put $R^{h}=0$ and replace $U_{0}^{h}, U_{1}^{h}, U_{2}^{h}$ by $V_{0}^{h}, V_{1}^{h}, V_{2}^{h}$, respectively. Finally, we obtain the system of equations

$$
\begin{aligned}
& B_{00} V_{0}^{h}+B_{01} V_{1}^{h}+B_{02} V_{2}^{h}=F_{0}^{h}+h^{2} A_{03} F^{h}, \\
& B_{10} V_{0}^{h}+B_{11} V_{1}^{h}+B_{12} V_{2}^{h}=F_{1}^{h}-F_{3}^{h}+h^{2}\left(A_{13}-A_{33}\right) F^{h}, \\
& B_{20} V_{0}^{h}+B_{21} V_{1}^{h}+B_{22} V_{2}^{h}=F_{2}^{h}-F_{3}^{h}+h^{2}\left(A_{23}-A_{33}\right) F^{h}
\end{aligned}
$$

for the new unknowns. Once system (22)-(24) has been solved, $v_{z z}^{h}\left(\omega_{i, j, k}\right)$ can readily be determined from (21).

Notice that the choice of the unknowns to be eliminated is relatively arbitrary. In a similar way we can eliminate $u_{x x}^{h}$ or $u_{y y}^{h}$ instead of $u_{z z}^{h}$.

\section{AN AUXILIARY FINITE ELEMENT}

On a "reference" cell $\hat{e}=[0,1]^{3}$ with the vertices $\hat{a}_{i}, i=1, \ldots, 8$ (Fig.2), consider the functions

$$
\hat{\theta}_{i}^{0}=\hat{\varphi}_{i}^{0}, \quad \hat{\theta}_{i}^{1}=\hat{\varphi}_{i}^{1}-\hat{\varphi}_{i}^{3}, \quad \hat{\theta}_{i}^{2}=\hat{\varphi}_{i}^{2}-\hat{\varphi}_{i}^{3}, \quad i=1, \ldots, 8
$$

where $\hat{\varphi}_{i}^{\alpha}, \alpha=0, \ldots, 3, i=1, \ldots, 8$ are defined by (2). Since functions (2) form the Lagrange basis for element (1), they are linearly independent. Hence, functions (25) are linearly independent as well and we can construct the space

$$
\hat{P}^{\prime}=\operatorname{span}\left\{\hat{\theta}_{i}^{\alpha}, \alpha=0,1,2, i=1, \ldots, 8\right\}
$$

of polynomials such that $\operatorname{dim} \hat{P}^{\prime}=24$.

Then we specify the set of DoF

$$
\hat{\Sigma}^{\prime}=\left\{\hat{\psi}_{i}^{0}(p)=p\left(a_{i}\right), \hat{\psi}_{i}^{1}(p)=p_{x x}\left(a_{i}\right), \hat{\psi}_{i}^{2}(p)=p_{y y}\left(a_{i}\right), i=1, \ldots, 8, \forall p \in \hat{P}^{\prime}\right\} .
$$

Observe that $\operatorname{card} \hat{\Sigma}^{\prime}=\operatorname{dim} \hat{P}^{\prime}$.

Direct calculations show that

$$
\hat{\psi}_{i}^{\alpha}\left(\hat{\theta}_{j}^{\beta}\right)=\left\{\begin{array}{l}
1 \text { for } \alpha=\beta, i=j \\
0 \text { otherwise }
\end{array}\right.
$$

i.e., functions (25) form the Lagrange basis in space (26) with respect to set (27) of DoF. Thus, the triple $\left(\hat{e}, \hat{P}^{\prime}, \hat{\Sigma}^{\prime}\right)$ is a finite element, which is shown in Fig. 2. 


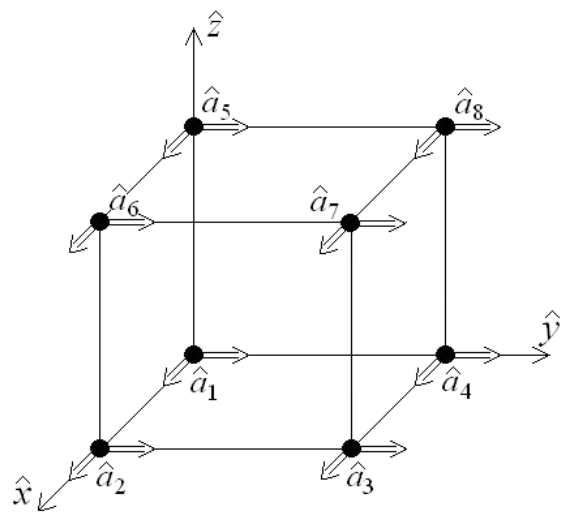

FIGURE 2. An auxiliary finite element.

The matrix of the system of equations of the finite element method for problem (4)-(5) constructed on the partition $\mathscr{T}_{h}$ with the auxiliary finite element can be written as

$$
A^{\prime}=\left(\begin{array}{ccc}
A_{00}^{\prime} & A_{01}^{\prime} & A_{02}^{\prime} \\
A_{10}^{\prime} & A_{11}^{\prime} & A_{12}^{\prime} \\
A_{20}^{\prime} & A_{21}^{\prime} & A_{22}^{\prime}
\end{array}\right)
$$

where $A_{\alpha, \beta}^{\prime}, \alpha, \beta=0,1,2$ are $N_{\text {int }} \times N_{\text {int }}$ blocks with the entries

$$
\left(A_{\alpha, \beta}^{\prime}\right)_{i, j, k, p, q, s}=a\left(\theta_{p, q, s}^{\beta}, \theta_{i, j, k}^{\alpha}\right) \quad i, j, k, p, q, s=1, \ldots, N-1
$$

The bilinear form $a$ is given by (9), the basis functions $\theta_{i, j, k}^{\alpha}$ of the finite element space are obtained from (25) with the help of an affine mapping of the form (3).

It is easy to verify that matrix (28) coincides with the matrix $B$ of system (22)-(24). In other words, the matrix of reduced system (22)-(24) is a matrix of a system of the finite element method but for elements with a smaller number of DoF. Hence, it is nonsingular and system (22)-(24) has a unique solution. Besides, the entries of the matrix of (22)-(24) can be immediately calculated as those of (28). This approach is more efficient in comparison with the manipulations with original matrix (7).

Of course, we can construct a discrete system for the auxiliary element, which differs from (22)-(24) by the righthand side, and obtain an approximate solution of (4)-(5). However, since for (26) we have the inclusion $\hat{P}^{\prime} \supset P$ only, this approach provides the same order of convergence as in the case of the usual trilinear element. Hence, at each node we have 2 redundant DoF. Thus, this element is not of practical importance in itself, but it is a useful tool when applying finite element (1) of higher degree.

\section{NUMERICAL RESULTS}

Let $\Omega=(0,1)^{3}$ and $\Gamma=\cup_{i=1}^{6} \Gamma_{i}$ be the boundary of $\Omega$ (Fig. 3). Consider the boundary value problem

$$
\begin{array}{ll}
-\Delta u=f \quad \text { in } \Omega, & \\
u=0 & \text { on } \Gamma_{1} \cup \Gamma_{2} \cup \Gamma_{3}, \\
u=y z \sin (y+z) & \text { on } \Gamma_{4}, \\
u=x z \sin (x+z) & \text { on } \Gamma_{5}, \\
u=x y \sin (x+y) & \text { on } \Gamma_{6}
\end{array}
$$

where $f=3 x y z \sin (1-x-y-z)+2(x y+y z+x z) \cos (1-x-y-z)$. The exact solution of (29)-(30) is of the form

$$
u=x y z \sin (1-x-y-z)
$$




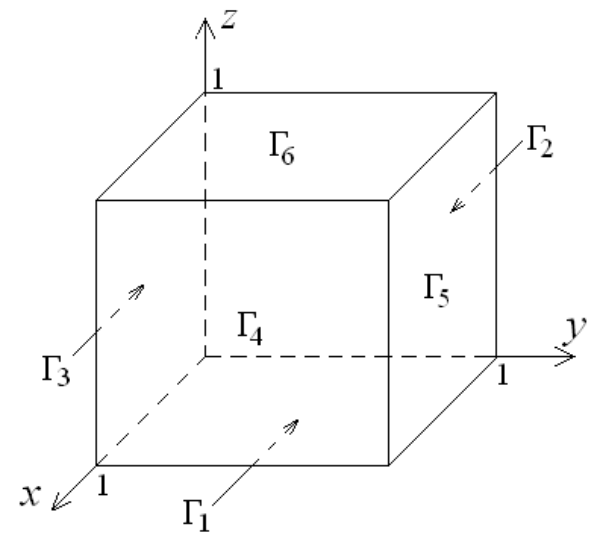

FIGURE 3. The domain $\Omega$ with the boundary $\Gamma$.

We subdivide $\Omega$ into elementary cubes for mash size $h=1 / N$. In numerical experiments we use $N=5,10,20,40$. On the obtained partition, along with standard system (10)-(13) for finite element (1), we construct and solve reduced system (22)-(24). The matrix of (22)-(24) is constructed using basis functions (25) of the auxiliary element.

To calculate the entries of the matrix and the components of the right-hand side vector for both systems, we apply the three-dimensional version of the Gauss-Lobatto quadrature rule [14] to provide high order accuracy of the method.

Since exact solution (31) is known, the error $u\left(\omega_{i, j, k}\right)-u^{h}\left(\omega_{i, j, k}\right)$ or $u\left(\omega_{i, j, k}\right)-v^{h}\left(\omega_{i, j, k}\right)$ can be calculated explicitly. Besides, we can calculate $\partial^{2} u / \partial x^{2}\left(\omega_{i, j, k}\right)-u_{x x}^{h}\left(\omega_{i, j, k}\right), \partial^{2} u / \partial y^{2}\left(\omega_{i, j, k}\right)-u_{y y}^{h}\left(\omega_{i, j, k}\right), \partial^{2} u / \partial z^{2}\left(\omega_{i, j, k}\right)-u_{z z}^{h}\left(\omega_{i, j, k}\right)$, $\partial^{2} u / \partial x^{2}\left(\omega_{i, j, k}\right)-v_{x x}^{h}\left(\omega_{i, j, k}\right), \partial^{2} u / \partial y^{2}\left(\omega_{i, j, k}\right)-v_{y y}^{h}\left(\omega_{i, j, k}\right)$, and $\partial^{2} v / \partial z^{2}\left(\omega_{i, j, k}\right)-u_{z z}^{h}\left(\omega_{i, j, k}\right)$ to estimate convergence of the second-order derivatives. We use the discrete norms

$$
\begin{aligned}
\left\|u-u^{h}\right\|_{h} & =h^{3 / 2}\left(\sum_{i, j, k=1}^{N-1}\left(u\left(\omega_{i, j, k}\right)-u^{h}\left(\omega_{i, j, k}\right)\right)^{2}\right)^{1 / 2}, \\
\left\|u-u^{h}\right\|_{H} & =h^{3 / 2}\left(\sum _ { i , j , k = 1 } ^ { N - 1 } \left(\left(u\left(\omega_{i, j, k}\right)-u^{h}\left(\omega_{i, j, k}\right)\right)^{2}+h^{4}\left(\frac{\partial^{2} u}{\partial x^{2}}\left(\omega_{i, j, k}\right)-u_{x x}^{h}\left(\omega_{i, j, k}\right)\right)^{2}\right.\right. \\
& \left.\left.+h^{4}\left(\frac{\partial^{2} u}{\partial y^{2}}\left(\omega_{i, j, k}\right)-u_{y y}^{h}\left(\omega_{i, j, k}\right)\right)^{2}+h^{4}\left(\frac{\partial^{2} u}{\partial z^{2}}\left(\omega_{i, j, k}\right)-u_{z z}^{h}\left(\omega_{i, j, k}\right)\right)^{2}\right)\right)^{1 / 2} .
\end{aligned}
$$

In Tables I-II we present results of the calculations for the standard system of the finite element method. We use the following notations:

$$
\varepsilon^{H}\left(u^{h}\right)=\left\|u-u^{h}\right\|_{H}, \quad \sigma_{x}^{h}\left(u^{h}\right)=\left\|\frac{\partial^{2} u}{\partial x^{2}}-u_{x x}^{h}\right\|_{h}, \quad \sigma_{y}^{h}\left(u^{h}\right)=\left\|\frac{\partial^{2} u}{\partial y^{2}}-u_{y y}^{h}\right\|_{h}, \quad \sigma_{z}^{h}\left(u^{h}\right)=\left\|\frac{\partial^{2} u}{\partial z^{2}}-u_{z z}^{h}\right\|_{h},
$$

$N_{u n}$ is the number of unknowns.

TABLE I. Accuracy of the approximate solution for the standard system.

\begin{tabular}{clcccc}
\hline \hline$N_{u n}$ & $h$ & $\varepsilon^{H}\left(u^{h}\right)$ & $\sigma_{x}^{h}\left(u^{h}\right)$ & $\sigma_{y}^{h}\left(u^{h}\right)$ & $\sigma_{z}^{h}\left(u^{h}\right)$ \\
\hline 500 & 0.2 & $5.94 \mathrm{E}-04$ & $1.23 \mathrm{E}-02$ & $1.23 \mathrm{E}-02$ & $1.23 \mathrm{E}-02$ \\
4000 & 0.1 & $5.02 \mathrm{E}-05$ & $3.49 \mathrm{E}-03$ & $3.49 \mathrm{E}-03$ & $3.49 \mathrm{E}-03$ \\
32000 & 0.05 & $3.62 \mathrm{E}-06$ & $9.17 \mathrm{E}-04$ & $9.17 \mathrm{E}-04$ & $9.17 \mathrm{E}-04$ \\
256000 & 0.025 & $2.44 \mathrm{E}-07$ & $2.36 \mathrm{E}-04$ & $2.36 \mathrm{E}-04$ & $2.36 \mathrm{E}-04$ \\
\hline \hline
\end{tabular}

Observe that the error $\varepsilon^{H}\left(u^{h}\right)$ tends to be of order 4 as $h$ decreases while $\sigma_{x}^{h}\left(u^{h}\right), \sigma_{y}^{h}\left(u^{h}\right)$, and $\sigma_{z}^{h}\left(u^{h}\right)$ tend to be of order 2. This is typical for cubic finite elements. 
TABLE II. A numerical convergence estimate for the standard system.

\begin{tabular}{ccccc}
\hline \hline$h$ & $\log _{2}\left(\varepsilon^{H}\left(u^{2 h}\right) / \varepsilon^{H}\left(u^{h}\right)\right)$ & $\log _{2}\left(\sigma_{x}^{h}\left(u^{2 h}\right) / \sigma_{x}^{h}\left(u^{h}\right)\right)$ & $\log _{2}\left(\sigma_{y}^{h}\left(u^{2 h}\right) / \sigma_{y}^{h}\left(u^{h}\right)\right)$ & $\log _{2}\left(\sigma_{z}^{h}\left(u^{2 h}\right) / \sigma_{z}^{h}\left(u^{h}\right)\right)$ \\
\hline \hline 0.1 & 3.56 & 1.81 & 1.81 & 1.81 \\
0.05 & 3.80 & 1.93 & 1.93 & 1.93 \\
0.025 & 3.89 & 1.96 & 1.96 & 1.96 \\
\hline \hline
\end{tabular}

Another series of calculations is related to the solution of the reduced system for the unknowns $v^{h}\left(\omega_{i, j, k}\right), v_{x x}^{h}\left(\omega_{i, j, k}\right)$, and $v_{y y}^{h}\left(\omega_{i, j, k}\right)$. In this case the values of $v_{z z}^{h}\left(\omega_{i, j, k}\right)$ are determined from (21). The results of calculations are presented in Tables III-IV.

TABLE III. Accuracy of the approximate solution for the reduced system.

\begin{tabular}{clcccc}
\hline \hline$N_{u n}$ & $h$ & $\varepsilon^{H}\left(v^{h}\right)$ & $\sigma_{x}^{h}\left(v^{h}\right)$ & $\sigma_{y}^{h}\left(v^{h}\right)$ & $\sigma_{z}^{h}\left(v^{h}\right)$ \\
\hline 375 & 0.2 & $1.38 \mathrm{E}-04$ & $2.87 \mathrm{E}-03$ & $2.87 \mathrm{E}-03$ & $2.87 \mathrm{E}-03$ \\
3000 & 0.1 & $1.21 \mathrm{E}-05$ & $8.41 \mathrm{E}-04$ & $8.41 \mathrm{E}-04$ & $8.41 \mathrm{E}-04$ \\
24000 & 0.05 & $8.86 \mathrm{E}-07$ & $2.25 \mathrm{E}-04$ & $2.25 \mathrm{E}-04$ & $2.25 \mathrm{E}-04$ \\
192000 & 0.025 & $5.98 \mathrm{E}-08$ & $5.81 \mathrm{E}-05$ & $5.81 \mathrm{E}-05$ & $5.80 \mathrm{E}-05$ \\
\hline \hline
\end{tabular}

TABLE IV. A numerical convergence estimate for the reduced system.

\begin{tabular}{ccccc}
\hline \hline$h$ & $\log _{2}\left(\varepsilon^{H}\left(v^{2 h}\right) / \varepsilon^{H}\left(v^{h}\right)\right)$ & $\log _{2}\left(\sigma_{x}^{h}\left(v^{2 h}\right) / \sigma_{x}^{h}\left(v^{h}\right)\right)$ & $\log _{2}\left(\sigma_{y}^{h}\left(v^{2 h}\right) / \sigma_{y}^{h}\left(v^{h}\right)\right)$ & $\log _{2}\left(\sigma_{z}^{h}\left(v^{2 h}\right) / \sigma_{z}^{h}\left(v^{h}\right)\right)$ \\
\hline \hline 0.1 & 3.52 & 1.77 & 1.77 & 1.77 \\
0.05 & 3.77 & 1.90 & 1.90 & 1.90 \\
0.025 & 3.89 & 1.96 & 1.96 & 1.96 \\
\hline \hline
\end{tabular}

Notice that the errors for $v^{h}$ are close to those for $u^{h}$. Moreover, in the case of the reduced system the accuracy is slightly better. Similar results were observed for two-dimensional problems [10, 11].

Thus, numerical experiments demonstrate the advantage of the proposed approach, namely, that the application of collocation enables one to reduce the number of unknowns without deterioration of the convergence order.

\section{ACKNOWLEDGMENTS}

The work is supported by Project 17-01-00270 of Russian Foundation for Basic Research.

\section{REFERENCES}

1. P. Ciarlet, The Finite Element Method for Elliptic Problems (North Holland, Amsterdam, Netherlands, 1978) p. 320.

2. F. K. Bogner, R. L. Fox, and L. A. Schmit, "The generation of interelement compatible stiffness and mass matrices by the use of interpolation formulas," Proceedings of the Conference on Matrix Methods in Structural Mechanics , 397-444 (1965).

3. S. Zhang, "On the full $c_{1}-q_{k}$ finite element spaces on rectangles and cuboids," Adv. Appl. Math. Mech. 2(6), 701-721 (2010).

4. S. Gopalacharyulu, "A higher order conforming, rectangular plate element," Int. J. Numer. Methods Engrg. 6, 305-308 (1973).

5. J. H. Argyris, I. Fried, and D. W. Scharpf, "The tuba family of plate elements for the matrix displacement method," Aero. J. Roy. Aero. Soc. 72, 514-517 (1968)

6. A. Zenisek, "Polynomial approximation on tetrahedrons in the finite element method," J. Approximation Theory 7, 334-351 (1973).

7. S. Zhang, "A family of 3d continuously differentiable finite elements on tetrahedral grids," Appl. Numer. Math. 59(1), 219-233 (2009).

8. N. J. Walkington, "A $c_{1}$ tetrahedral finite element without edge degrees of freedom," SIAM J. Numer. Anal. 52(1), 330-342 (2014).

9. V. Shaidurov, S. Shut, and L. Gileva, "Some properties of hermite finite elements on rectangles," in Application of Mathematics In Technical and Natural Sciences: 6th International Conference for Promoting the Application of Mathematics in Technical and Natural Sciences AMiTaNS '14, AIP Conference Proceedings, Vol. 1629, edited by M. D. Todorov (AIP Publishing, NY, USA, 2014) pp. 32-43.

10. L. Gileva, E. Karepova, and V. Shaidurov, "The application of a special hermite finite element coupled with collocation to the diffusion equation," (Springer, 2018) pp. 44-55. 
11. L. Gileva, E. Karepova, and V. Shaidurov, "A combination of a special hermite finite element with collocation for a reaction-diffusion type equation,” Lobachevskii J. Math. 40(4), 471-480 (2019).

12. R. Adams and I. Fournier, Sobolev Spaces (Academic Press, NY, USA, 2003) p. 320.

13. S. Brenner and L. Scott, The Mathematical Theory of Finite Element Method (Springer-Verlag, Berlin-Heidelberg-New York, 1994) p. 420.

14. M. Abramowitz and I. A. Stegan, Handbook of Mathematical Functions with Formulas with Formulas, Graphs, and Mathematical Tables (U.S. Department of Commerce, Natl. Bureau of Standards, New York, 1973). 